\title{
Lipid Nanocapsule as Vaccine Carriers for His-Tagged Proteins: Evaluation of Antigen-Specific Immune Responses to HIV I His- Gag p41 and Systemic Inflammatory Responses
}

\author{
Saurabh Wadhwa ${ }^{1}$, Anekant Jain ${ }^{1}$, Jerold G. Woodward ${ }^{2}$, and Russell J Mumper ${ }^{*}, 1,3,4$ \\ ${ }^{1}$ Division of Molecular Pharmaceutics, UNC Eshelman School of Pharmacy, University of North \\ Carolina at Chapel Hill, NC 27599-7362 \\ ${ }^{2}$ Department of Microbiology, Immunology and Molecular Genetics, University of Kentucky, \\ Lexington, KY 40506 \\ ${ }^{3}$ Center for Nanotechnology in Drug Delivery, University of North Carolina at Chapel Hill, NC \\ 27599-7362 \\ ${ }^{4}$ UNC Lineberger Comprehensive Cancer Center, University of North Carolina at Chapel Hill, NC \\ 27599-7295
}

\begin{abstract}
The purpose of this study was to design novel nanocapsules (NCs) with surface-chelated nickel (Ni-NCs) as a vaccine delivery system for histidine (His)-tagged protein antigens. Ni-NCs were characterized for binding His-tagged model proteins through high affinity non-covalent interactions. The mean diameter and zeta potential of the optimized Ni-NCs was $214.9 \mathrm{~nm}$ and $14.8 \mathrm{mV}$, respectively. The optimal binding ratio of His-tagged Green Fluorescent Protein (HisGFP) and His-tagged HIV-1 Gag p41 (His-Gag p41) to the Ni-NCs was 1:221 and 1:480 w/w, respectively. Treatment of DC2.4 cells with Ni-NCs did not result in significant loss in the cell viability up to $24 \mathrm{~h}(<5 \%)$. We further evaluated the antibody response of the Ni-NCs using HisGag p41 as a model antigen. Formulations were administered subcutaneously to BALB/c mice at day 0 (prime) and 14 (boost) followed by serum collection on day 28. Serum His-Gag p41 specific antibody levels were found to be significantly higher at 1 and $0.5 \mu \mathrm{g}$ doses of Gag p41-His-NiNCs (His-Gag p41 equivalent) compared to His-Gag p41 (1 $\mu \mathrm{g})$ adjuvanted with aluminum hydroxide (AH). The serum IgG2a levels induced by Gag p41-His-Ni-NCs $(1 \mu \mathrm{g})$ were significantly higher than AH adjuvanted His-Gag p41. The Ni-NCs alone did not result in elevation of systemic IL-12/p40 and CCL5/RANTES inflammatory cytokine levels upon subcutaneous administration in BALB/c mice. In conclusion, the proposed Ni-NCs can bind Histagged proteins and have the potential to be used as antigen delivery system capable of generating strong antigen specific antibodies at doses much lower than with aluminum based adjuvant and causing no significant elevation of systemic proinflammatory IL-12/p40 and CCL5/RANTES cytokines.
\end{abstract}

\footnotetext{
(C) 2011 Elsevier B.V. All rights reserved.

*Corresponding Author: John A. McNeill Distinguished Professor Director, Center for Nanotechnology in Drug Delivery Division of Molecular Pharmaceutics UNC Eshelman School of Pharmacy CB 7355, 100G Beard Hall University of North Carolina at Chapel Hill Chapel Hill, North Carolina 27599-7355 mumper@email.unc.edu Phone: (919) 966-1271 Fax: (919) 966-6919.

Publisher's Disclaimer: This is a PDF file of an unedited manuscript that has been accepted for publication. As a service to our customers we are providing this early version of the manuscript. The manuscript will undergo copyediting, typesetting, and review of the resulting proof before it is published in its final citable form. Please note that during the production process errors may be discovered which could affect the content, and all legal disclaimers that apply to the journal pertain.
} 


\section{Keywords}

Green Fluorescent Protein; antigen; dendritic cells; adjuvant; cytokine; antibody

\section{Introduction}

Subunit vaccines containing soluble protein antigens have been proposed as alternatives to using whole organisms to generate immune responses because of reproducible immune responses and better characterization [1]. However, many of these antigens are poorly immunogenic if administered alone. Particulate antigen delivery systems like nanoparticles (NPs), emulsions, microparticles and liposomes have been shown to enhance the recognition of the antigens by the antigen presenting cells (APCs) and result in improved immune response [2-5]. Formation of a depot at the site of injection has been proposed as a possible mechanism of enhanced antigen recognition by particulate systems [6]. In addition to being taken up efficiently by the APCs, NPs have the potential to release the entrapped antigen over prolonged time. Moreover, surface modification allows incorporation of a variety of antigens on the same particle and surface-coated ligands to target the APCs $[7,8]$.

The antigen can be either entrapped inside the matrix/core of the particle or coated on the surface. The entrapment of protein in the core of a particle has problems associated with the stability of the protein during the preparation of particles and poor entrapment [9]. The surface coating of the antigen on the particle may be achieved by ionic interactions [10], covalent conjugation [11] or non-covalent attachment [12]. We have previously reported that increasing the affinity of the antigen to the surface of solid lipid NPs contributes to enhanced immune responses as compared to the antigens associated on the surface via simple adsorption or charge-charge interactions [10, 12].

In the present study, we investigated the formulation of novel nanocapsules (NCs) with surface chelated nickel (Ni-NCs) in the outer shell and their potential to bind histidine (His)tagged proteins with high affinity through non-covalent attachment. The strong non covalent interaction $\left(\mathrm{K}_{\mathrm{D}} \sim 10^{-6}-10^{-14} \mathrm{M}\right)$ between affinity ligands such as the His-tag on a protein and transition metal ions like $\mathrm{Ni}$ and $\mathrm{Cu}$ has been investigated in detail and has been successfully applied to protein purification [13-15]. This interaction is highly dependent on individual protein, the site and length of His-tag and $\mathrm{pH}[14,16]$. Liposomes and iron oxide NPs with surface chelated $\mathrm{Ni}$ have been previously proposed for the delivery and purification of His-tagged proteins [17, 18]. We formulated the Ni-NCs using 1,2-dioleoyl$s n$-glycero-3-[(N-(5-amino-1-carboxypentyl) iminodiacetic acid) succinyl] (nickel salt) (DGS-NTA-Ni), a lipid with NTA end group that can chelate Ni. Platt and co-workers concluded that the association of NTA-Ni and his-tagged proteins is in micromolar range which may be considered weak for in-vivo conditions. However, later studies comparing NTA to trivalent NTA ligands suggested that increasing the affinity of this interaction did not lead to an increase in immune responses [18, 19]. Although this report showed that covalently bound antigen elicits stronger responses, the effect of the nature of association on immune response may be antigen-specific as was reported by Shahum and Therien [20]. Moreover, it has been previously known that covalent modification of antigens is prone to causing changes in the antigenicity and loss of binding [21]. Non-covalent attachment while enhancing the antigen association is expected to preserve the antigenicity by ensuring the presentation of the unmodified antigen.

Aluminum salts remain the only FDA approved particulate adjuvants. They have been shown to induce strong antibody responses but there is uncertainty in induction of cellular immunity [22]. Additionally, the use of aluminum containing salts has been linked to 
hypersensitivity reactions and physical or chemical alterations of the adsorbed protein antigen in some cases [23]. NPs have been investigated for their superior safety profile and an ability to protect the entrapped antigen [24]. In addition, we have reported strong humoral and cellular immune responses against several protein antigens like TAT, p24 and Nef coated onto solid lipid NPs and that NP bound antigens have the potential to generate CD8+ $\mathrm{T}$ cell responses [25].

In the present studies, we investigated a new type of lipid-based NCs developed in our laboratory for their potential to deliver His-tagged proteins. His-Gag p41 was used as a model antigen. We also compared the immune responses from our previously reported [12] nickel decorated solid lipid NPs (Ni-NPs) to the novel Ni-NCs.

\section{Materials and Methods}

\subsection{Materials and Reagents}

Polyoxyethylene (20) stearyl ether (Brij® 78), d- $\alpha$-tocopheryl polyethylene glycol 1000 succinate (Vitamin E TPGS) and Miglyol® 812 (caprylic/capric triglycerides) were purchased from Uniqema (Wilmington, DE), Eastman Chemicals (Kingsport, TN) and Sasol (Witten, Germany), respectively. Sepharose ${ }^{\circledR}$ CL-4B and DGS-NTA-Ni were obtained from GE Healthcare (Piscataway, NJ) and Avanti Polar Lipids (Alabaster, AL), respectively. Inductively Coupled Plasma-Mass Spectrometry (ICP-MS) standard for Nickel was purchased from Sigma Aldrich (St. Louis, MO) and N-terminal His-tagged GFP (His-GFP) was purchased from Millipore (Billerica, MA). Aluminum hydroxide gel (Cat. No. AL226) and emulsifying wax (comprised of cetyl alcohol and polysorbate 60 in a molar ratio of 20:1) were purchased from Spectrum Chemicals (Gardena, CA). CpG oligonucleotide (5'tcc atg acg ttc ctg acg tt $\left.-3^{\prime}\right)$ (20 mer) (CpG ODN) was purchased from InvivoGen (San Diego, CA).

\subsection{Preparation of Ni-NCs}

To prepare Ni-NCs, Brij 78 (3.5 mg), Vitamin E TPGS (1.5 mg) and Miglyol 812 (2.5 mg) were weighed in a glass vial. DGS-NTA-Ni (varying amounts of $10 \mathrm{mg} / \mathrm{mL}$ stock solution in chloroform) and $0.2 \mathrm{~mL}$ ethanol were added and mixed. The solvents were later evaporated under nitrogen. The vial was placed in a water bath at $65^{\circ} \mathrm{C}$ and deionized water $(1 \mathrm{~mL})$ preheated to $65^{\circ} \mathrm{C}$ was added while stirring the contents for $30 \mathrm{~min}$. The Ni-NCs form spontaneously and are composed of liquid core (Miglyol 812) and solid shell (Brij 78 and Vitamin E TPGS). The suspension was cooled to room temperature and separated from free components using a Sepharose CL-4B column $(1.5 \times 15 \mathrm{~cm})$. The purified Ni-NCs were characterized for particle size using Beckman Coulter N5 Submicron Particle Size Analyzer (Beckman Coulter, Brea, CA) and zeta potential using a Malvern Nano-Z (Malvern Instruments, Southborough, MA). Formulations were prepared by adding $0.1 \mathrm{mg}$ (NC01), $0.25 \mathrm{mg}$ (NC02) or $0.5 \mathrm{mg}$ (NC03) DGS-NTA-Ni. A formulation with no DGS-NTA-Ni (NC00) was prepared and used as control. For comparison, Ni-NPs were prepared as previously reported [12]. Briefly, emulsifying wax $(2 \mathrm{mg})$ and Brij $78(3.5 \mathrm{mg})$ were weighed in a glass vial and heated to $65^{\circ} \mathrm{C}$. DGS-NTA-Ni $(0.106 \mathrm{mg} ; 10 \mathrm{mg} / \mathrm{mL}$ stock solution in chloroform) was added to the mixture. Deionized water $(1 \mathrm{~mL})$ was added and the contents were stirred at $65^{\circ} \mathrm{C}$ to form a clear oil-in-water microemulsion. Ni-NPs were obtained by cooling the microemulsion to room temperature that causes the solidification of the core and the shell components. The Ni-NPs were purified as described above.

\subsection{Determination of Surface Nickel Content}

ICP-MS was used to quantify the amount of $\mathrm{Ni}$ on the surface of the Ni-NCs available for binding to His-tagged ligands using Varian 820 Mass Spectrometer (Palo Alto, CA). A 
standard curve for Ni was prepared using Ni standard solution $(1000 \mathrm{mg} / \mathrm{L})$ in the concentration range of $9-200 \mathrm{ppb}$. The recovery of Ni from the Ni-NCs was quantified by spiking the standards with Ni-NCs dissolved in $0.2 \mathrm{~mL}$ of ethanol. To quantify the amount of Ni on the surface, the Ni-NCs were diluted with $2 \%$ nitric acid, filtered through a $0.2 \mu \mathrm{m}$ filter and analyzed by ICP-MS. Ni concentrations were calculated from the previously developed standard curve. Formulation $\mathrm{NC} 02$ was selected for further studies based on lower polydispersity and higher DGA-NTA-Ni incorporation (wt \%) compared to other formulations (Table 1).

\subsection{His-GFP Biding to Ni-NCs}

To investigate the extent of accessible $\mathrm{Ni}$ on the surface, formulation $\mathrm{NC} 02$ was mixed with His-GFP in several Ni to His-GFP molar ratios (1:0.1, 1:0.2, 1:0.4 and 1:0.8) and incubated at $4{ }^{\circ} \mathrm{C}$ overnight to allow for surface binding. Formulation NC00 was also incubated with His-GFP (amount similar to that used in the ratio 1:0.2 above) to determine non-specific association. The GFP-His-Ni-NC02 was separated from unbound His-GFP using a Sepharose CL-4B column $(1.5 \times 15 \mathrm{~cm})$ equilibrated with $10 \mathrm{mM}$ PBS. Briefly, $0.2 \mathrm{~mL}$ mixture was applied to the column and $1 \mathrm{~mL}$ fractions were collected. Subsequently, the fluorescence associated with each fraction was measured (Ex 360/40, Em 528/20) on a Synergy ${ }^{\text {TM }} 2$ Multi-Detection Microplate Reader (Biotek, Winooski, VT). The particle intensity of each fraction was also measured and fractions containing GFP-His-Ni-NC02 were pooled and characterized for their particle size and zeta potential, respectively.

\subsection{Microscopy}

Formulation NC02 was visualized by transmission electron microscopy (TEM) to understand the morphology of the particles. Briefly, $5 \mu \mathrm{L}$ (approximately $1.6 \times 10^{6}$ particles) of diluted NC02 suspension was spread on a Pelco formvar coated 300 mesh copper grid (01710-F, Ted Pella, Redding, CA). The suspension was allowed to air dry for 5 min. Any remaining liquid was wicked off. There was no additional staining. The grids were then examined with a Zeiss EM 900 Transmission Electron Microscope using $50 \mathrm{kV}$ accelerating voltage. The images were acquired using photographic film which was subsequently digitized.

\subsection{Effect of GFP-His-Ni-NCs on the Viability of Dendritic Cells}

The DC2.4 murine dendritic cell line was obtained from Dr. Kenneth L. Rock (Department of Pathology, University of Massachusetts Medical School). The cells were cultured in RPMI 1640 (ATCC) supplemented with 10\% fetal bovine serum, $2 \mathrm{mM}$ glutamine, $55 \mu \mathrm{M}$ beta-mercaptoethanol, non-essential amino acids, $10 \mathrm{mM}$ HEPES buffer and $100 \mathrm{U} / \mathrm{mL}$ penicillin and $100 \mu \mathrm{g} / \mathrm{mL}$ streptomycin (GIBCO). Trypan blue was used to determine viability and cells were used for experiments when 70-80\% confluent.

The DC2.4 dendritic cells were incubated with different concentrations of $\mathrm{NC} 02$ for up to 48 h. The cells were trypsinized with $0.05 \%$ Trypsin-EDTA (GIBCO), suspended in culture medium, centrifuged at $200 \mathrm{~g}$ x $5 \mathrm{~min}$ and re-suspended in $10 \mathrm{mM}$ PBS containing $1 \mathrm{mM}$ EDTA and $10 \%$ fetal bovine serum. Propidium iodide (PI) was added to the cell suspension as a viability marker at a final concentration of $1 \mu \mathrm{g} / \mathrm{mL}$ immediately before analysis by flow cytometry. Flow cytometric analysis was performed using BD LSRII Flow Cytometer (BD Biosciences, Mountain View, CA). Data analysis was performed using Flow Jo 7.6Software (Tree Star, Ashland, OR). 


\subsection{His-Gag p41 Binding to Ni-NCs}

Purified recombinant His-Gag p41 protein (HXB2 isolate) was generously provided by Dr. Robert Seder (NIH-NIAID) and was used as model antigen. The protein was fluorescently labeled using NHS-fluorescein. Briefly, $16 \mu \mathrm{g}$ of NHS-fluorescein $(186 \mu \mathrm{g} / \mathrm{mL}$ stock solution in DMSO) was added to $325 \mu \mathrm{g}$ of His-Gag p41 $(1.2 \mathrm{mg} / \mathrm{mL}$ stock solution in 10 $\mathrm{mM}$ PBS). The mixture was incubated overnight at $4^{\circ} \mathrm{C}$. Excess reactants were separated from the protein using a desalting spin column. The degree of modification was calculated using the molar extinction coefficient of fluorescein $\left(\varepsilon=68,000 \mathrm{M}^{-1} \mathrm{~cm}^{-1}, \lambda_{\max }=493 \mathrm{~nm}\right)$. The fluorescein-His-Gag p41 was incubated with $\mathrm{NC} 02$ in different weight ratios (His-Gag p41:NC02) as described previously for His-GFP to estimate the binding of fluorescein-HisGag p41 to NC02.

\subsection{Mouse Immunization Study}

Eight to 10-weeks old female BALB/c mice ( $\mathrm{n}=5-7 /$ group) from Charles River Laboratories were used for immunization studies. The experimental design is shown in Table 3. Formulations $(100 \mu \mathrm{L})$ were administered subcutaneously in the nape of the neck on day 0 and 14. Gag p41-His-Ni-NC02 (His-Gag p41:Ni-NCs weight ratio of 1:480) was used to prepare three different concentrations that were subsequently administered to mice to give three different doses of His-Gag p41. Gag p41-His-Ni-NP (His-Gag p41:Ni-NP weight ratio of 1:100) were administered for comparison. Immune responses were compared to His-Gag p41 adjuvanted with $\mathrm{AH}$. An aqueous suspension of $\mathrm{AH}(4.8 \mathrm{mg} / \mathrm{mL})$ was prepared in deionized water by ultrasonication and His-Gag p41 $(10 \mu \mathrm{g} / \mathrm{mL})$ was added prior to the injections. Mice were bled by cardiac puncture on day 28 and sera were collected. All sera were stored at $-20^{\circ} \mathrm{C}$ until further analysis.

\subsection{Determination of Antibody Levels}

His-Gag p41 specific serum IgG, IgG1 and $\operatorname{IgG} 2$ a antibody levels were determined by ELISA. Briefly, 96-well plates were coated with $50 \mu \mathrm{L}$ His-Gag p41 (5 $\mu \mathrm{g} / \mathrm{mL}$ in PBS) overnight at $4^{\circ} \mathrm{C}$. The wells were washed with PBS/Tween 20 and blocked using $4 \%$ BSA prepared in PBS/Tween 20 for $1 \mathrm{~h}$ at $37^{\circ} \mathrm{C}$. Subsequently, the wells were washed with PBS/ Tween 20 and $50 \mu \mathrm{L}$ of mouse serum at predetermined dilution was added and plates were incubated for $2 \mathrm{~h}$ at $37^{\circ} \mathrm{C}$. The wells were washed with PBS/Tween 20 and Horseradish Peroxidase (HRP) conjugated Anti-mouse $\operatorname{IgG~F}\left(\mathrm{ab}^{\prime}\right)_{2}$ fragment from sheep $(50 \mu \mathrm{L}$ of 1:3000 dilution in $1 \% \mathrm{BSA}$ in PBS/Tween 20) was added and incubated for $1 \mathrm{~h}$ at $37^{\circ} \mathrm{C}$. The wells were washed with PBS/Tween 20 and TMB (3,3,5,5-tetramethylbenzidine) substrate $(100 \mu \mathrm{L})$ was added to each well and incubated for $30 \mathrm{~min}$ at RT. Color development was stopped by addition of $2 \mathrm{M}$ sulfuric acid and the absorbance at $450 \mathrm{~nm}$ was measured on Synergy ${ }^{\mathrm{TM}} 2$ Multi-Detection Microplate Reader (Biotek, Vinooski, VT). For the determination of $\operatorname{IgG} 1$ and $\operatorname{IgG} 2 \mathrm{a}$, the plates were blocked for $1 \mathrm{~h}$ at $37^{\circ} \mathrm{C}$ as described above followed by incubation with mouse serum for $1 \mathrm{~h}$ at RT. The plates were incubated with biotinylated rat anti-mouse $\operatorname{IgG} 1$ or $\operatorname{IgG} 2 \mathrm{a}$ for $1 \mathrm{~h}$ at RT followed by incubation with streptavidin HRP for $30 \mathrm{~min}$ at RT. The plates were developed as described for IgG.

\subsection{Systemic Cytokine Induction Studies}

To investigate the induction of inflammatory cytokines upon s.c. administration of Ni-NCs, 8-10 weeks old female BALB/c mice ( $\mathrm{n}=3 /$ time point) were used. Briefly, $0.48 \mathrm{mg}$ of $\mathrm{NC} 02$ or $10 \mu \mathrm{g}$ CpG ODN $(0.1 \mathrm{mg} / \mathrm{mL}$ in PBS) were administered to mice. Blood was collected by cardiac puncture at $3,6,12,24$ and $48 \mathrm{~h}$ after administration. Sera were collected and stored at $-20^{\circ} \mathrm{C}$. The serum concentration of IL-12/p40 and RANTES/CCL5 were determined by $\mathrm{BD}^{\mathrm{TM}}$ cytometric bead array (CBA) flex assay following manufacturer's protocol (BD Pharmingen, La Jolla, CA). Flow cytometric analysis was performed using 
BD $^{\mathrm{TM}}$ LSRII Flow Cytometer (BD Biosciences, Mountain View, CA). Data analysis was performed using Flow Jo 7.6Software (Tree Star, Ashland, OR).

\subsection{Statistical Analysis}

Statistical analysis was performed using GraphPad Prism 4 Software (GraphPad Software, San Diego, CA). Differences in antibody responses among treatment groups were analyzed by one way ANOVA followed by Dunnet's post-test to compare different treatments to the naïve group. Effects on DC2.4 cell viability and cytokine induction results were analyzed by regular two-way ANOVA followed by Bonferroni post-test to compare differences within groups. Differences were considered significant at $\mathrm{p}<0.05$.

\section{Results}

\subsection{Preparation and Characterization of Ni-NCs}

Our laboratory has recently reported the formulation of stable spontaneously forming NCs containing a liquid oil core and surfactant-based shell prepared using biocompatible and biodegradable components [26,27]. The liquid core consists of Miglyol 812, which is a mixture of caprylic and capric acid triglycerides, while the shell is composed of two nonionic surfactants, Brij 78 and Vitamin E TPGS respectively. We modified the formulation to contain a small amount of surface accessible nickel by incorporating DGSNTA-Ni in the shell (Fig. 1a). DGS-NTA-Ni has two out of the six co-ordination sites available to interact with the His residues on proteins. Formulations prepared during the optimization of NCs with or without surface accessible nickel. The incorporation of DGSNTA-Ni was determined by measuring the amount of Ni by ICP-MS. Based on the incorporation efficiency of DGS-NTA-Ni determined by ICP-MS analysis and desired particle size range, formulation NC02 was selected for further studies. The optimized NCs had mean particle size of $214 \mathrm{~nm}$ with very low polydispersity upon gel filtration and had negative surface charge density as indicated by zeta potential measurements in $0.5 \mathrm{mM}$ phosphate buffer (Table 1). The negative charges can be due to the formation of small amounts of metal oxides or accumulation of negative ions on the surface of the NCs. It is interesting to note that the relationship between nickel incorporation and zeta potential is non-linear for NCs described in the present study. This may be explained by partial solubility of DGS-NTA-Ni in the core of NCs whereby the NTA-Ni entrapped in the core of NCs does not contribute to the zeta potential of the NPs. We also formulated previously reported Ni-NPs to investigate the effect of particle morphology on antigen delivery [12]. The Ni-NPs have a solid matrix core composed of emulsifying wax coated with a single surfactant.

\subsection{Protein Binding}

His-GFP was used as a model protein to study the interaction of His-tag with surface $\mathrm{Ni}$ and investigate the binding efficiency of the Ni-NCs. His-GFP can be easily detected by fluorescence making it easier to quantitatively determine the amount of protein bound to the surface and qualitative assessment by microscopy [28]. The GFP-His-Ni-NC02 was separated from unbound His-GFP using gel filtration (Sepharose CL-4B). The fluorescence associated with the particles compared to the fluorescence of the unbound fractions was used to derive the percent of His-GFP bound to the Ni-NCs (Fig. 2a). The elution of particles from the gel was monitored using particle intensity measurements by light scattering. Based on trials using different ratios of surface Ni to His-GFP, Ni-NCs (NC02-02) incubated at molar ratio of 1:0.2 (Ni:His-GFP) resulted in the highest level of binding (35\%). Interestingly, increasing the amount of GFP did not increase the association (Table 2). This indicates that only a limited number of DGS-NTA-Ni are accessible on the surface of the $\mathrm{NCs}$ and the binding is therefore, saturable. Theoretical calculations showed that the weight 
ratio His-GFP: Ni-NC in formulation NC02-02 was 1:221 and the ratio of molecules of $\mathrm{Ni}$ $(13,300)$ to molecules of His-GFP $(885)$ was approximately 15 . This is expected as the much larger surface area of His-GFP may result in shielding of the surface $\mathrm{Ni}$ and the ability of single His-tag residue to complex with multiple Ni atoms. The binding of protein on the surface did not significantly affect the particle size and zeta potential of Ni-NCs (data not shown). Formulation NC00 was used as controls to determine non-specific binding of HisGFP which was found to be negligible (Fig. 2a). The results from this study provide an evidence of surface binding of His-tagged proteins that was specific to Ni-NCs. The binding experiments were also performed using fluorescein-His-Gag p41. The weight ratio of 1:480 (His-Gag p41: NC02) resulted in more than $65 \%$ of the protein associated with the $\mathrm{NC02}$ (Fig. 2b).

\subsection{Microscopy}

To examine the morphology of the Ni-NCs, $\mathrm{NCO} 2$ suspension was observed by TEM. As the $\mathrm{Ni}-\mathrm{NCs}$ have a soft structure and all the components have lower melting points, the protocol involved air drying of the grids. The NCs appeared as hollow spheres that confirms the presence of a fluid core (Fig. 1b). This is in contrast to similar TEM studies performed with our previous solid lipid nanoparticles where the core is composed of waxy solid and the particles appear as opaque spheres [29]. The size observed by TEM was smaller than that measured by dynamic light scattering. The NCs of the present study have an internal fluid structure comprised of an aqueous-bound shell. This may contribute to shrinkage and collapse during drying and imaging.

\subsection{Toxicity of NCs in Dendritic Cells}

Dendritic cells are the most important APCs. Particulate delivery systems are suited for rapid uptake by the dendritic cells due to their size.The cytotoxicity of the Ni-NCs (NC02) to DCs following treatment with NCs for 12,24 or $48 \mathrm{~h}$ was investigated by flow cytometry (Fig. 3). At 12 and $24 \mathrm{~h}$, none of the concentrations tested resulted in $>5 \%$ increase in cytotoxicity over untreated cells. However, at $48 \mathrm{~h}$, the Ni-NC treatment resulted in significant cytotoxicity to DCs. In our previous studies, we have reported significant cytotoxicity in DCs treated with lower concentrations of Ni-NPs $(11 \mu \mathrm{g} / \mathrm{mL})$ [8]. In-vivo, this difference may result in Ni-NCs being better tolerated compared to Ni-NPs making it possible to dose higher concentrations of protein-bound NCs.

\subsection{Immunization Study}

Mice were immunized with three different doses of His-Gag p41/Ni-NCs (fixed weight ratio of 1:480) to investigate a His-Gag p41 dose response in the antibody generation. Comparisons were made with His-Gag p41 adjuvanted with AH. At 1:1000 sera dilution, the $\mathrm{Ni}-\mathrm{NCs}$ resulted in stronger antibody response compared to $\mathrm{AH}$ at both the $1 \mu \mathrm{g}$ and $0.5 \mu \mathrm{g}$ His-Gag p41 doses (Fig. 4a). When compared at the same sera dilution, the OD $(450 \mathrm{~nm})$ values for NCs at the $1 \mu \mathrm{g}$ dose were $>4$-fold higher than those for Ni-NPs. As discussed above, it is possible that the toxicity to APCs may have contributed to weaker antibody response observed with the Ni-NPs.

The serum isotype levels, $\operatorname{IgG} 1$ and $\operatorname{IgG} 2 \mathrm{a}$, were determined by ELISA to investigate the type of immune response at 1:500 serum dilution for all the formulations. While production of $\operatorname{IgG} 2 \mathrm{a}$ isotype has been associated with Th1 response, $\operatorname{IgG1}$ isotype has been associated with Th2 type response. The ratios of $\operatorname{IgG} 2 \mathrm{a} / \mathrm{IgG} 1$ were used to indicate the Th1 or Th2 bias of the generated immune response [30, 31]. A balanced Th1 and Th2 response is desired following vaccination as excess of either will result in adverse effects. Aluminum containing salts have been reported to generate a Th2 biased response [32,33]. This is expected to 
result in a lower $\operatorname{IgG} 2 \mathrm{a} / \mathrm{IgG} 1$ ratio. In our observations, the ratio $\mathrm{IgG} 2 \mathrm{a} / \mathrm{IgG} 1$ was 0.14 for $\mathrm{AH}$ and 0.55 for Ni-NCs at highest dose of His-Gag p41 $(1 \mu \mathrm{g})$ respectively (Fig. $4 \mathrm{~b})$.

\subsection{Systemic Cytokine Induction Studies}

To investigate the immunostimulating activity of Ni-NCs, we determined the induction of pro-inflammatory cytokines by Ni-NCs (formulation NC02). CpG ODN was administered as strong inducer of pro-inflammatory cytokines. We determined serum concentrations of IL-12/p40 and CCL5/RANTES upon s.c. injection of Ni-NCs in comparison to CpG ODN and untreated animals. The results demonstrate that Ni-NCs did not result in significant elevation in the level of any of the cytokines measured over control while $\mathrm{CpG}$ was a strong inducer of both the cytokines (Fig. 5). This indicates that the Ni-NCs are potentially a safe delivery vehicle and may result in fewer adverse events upon administration. However, this also indicates that additional (safe) adjuvant(s) may be needed in certain cases to potentiate the immune response by Ni-NCs.

\section{Discussion}

This study aimed at developing NCs with chelated Ni on the surface thus providing binding sites for His-tagged antigens. The Ni-NCs of the present study are composed of completely metabolizable or biocompatible components and have the ability to carry multiple antigens as well as an adjuvant. Therefore, using them as a vehicle for the delivery of vaccines is a promising approach.

Delivery of antigen by particulate systems requires their association or entrapment within the particle. The effect of the type of association on the resulting immune response has been a matter of debate over the past two decades and several contradicting reports have been published $[19,20]$. In our previous studies, we observed that increasing the association of the antigen to the surface of the NPs increased the associated antibody responses [12]. Continuing on the previously obtained results, we investigated non-covalent attachment of the antigen to the NPs exploiting the Ni-His interaction. NTA has been used as a popular ligand for $\mathrm{Ni}$ in delivery systems [34]. The effect of spacer group and the valency show that while the length of the spacer may have some effect on the accessibility, the valency does not affect the antibody responses $[19,35]$. The Ni-NCs exhibited specific binding ability for His-tagged model protein although the extent of binding can be increased by improving the binding affinity of surface nickel. This may be done by modifying the length and amphiphilicity of the spacer group to reduce steric effects on the surface of the NPs although some steric hindrance is desired to reduce the exchange of the bound protein with other Histagged proteins or serum proteins.

The overall safety and absence of hypersensitivity are desirable features of a vaccine formulation. The presence of $\mathrm{Ni}$ in the formulations of the present study may be of concern in view of studies implicating adverse events like immunosuppression [36] and carcinogenicity [37] upon parenteral administration of $\mathrm{Ni}$ compounds and $\mathrm{Ni}$ containing products. However, the highest dose of the $\mathrm{Ni}$ administered in the form of Ni-NCs in the present study was $70 \mathrm{ng}$. This is approximately 10,000-fold less than the doses shown to cause immunosuppression or carcinogenicity and is expected to be well tolerated. Additionally, the average dietary intake of $\mathrm{Ni}$ in humans is estimated to be 69-162 $\mu \mathrm{g} / \mathrm{day}$ (Safe Use of Nickel, 2008). Chikh et al. reported no toxicity upon acute s.c. dosing (three weekly injections) of liposomes containing 5\% and 10\% DGS-NTA-Ni in mice for 30 days [38]. To further address this, we examined the serum levels of IL-12/p40 and CCL5/ RANTES in mice treated with Ni-NCs. We observed no significant induction of systemic pro-inflammatory cytokines by s.c. administered Ni-NCs. Nevertheless, further investigation of immune response to DGS-NTA-Ni may be needed and antigen delivery systems using 
components containing Ni must be evaluated for their safety in individuals where adverse events may be expected.

The type of desired immune response, humoral or cellular, is affected by many factors including the nature of the pathogen or the antigen, its mode of entry, types of cells infected and cellular localization, the purpose of immune induction (therapeutic or prophylactic) and host features among others. In most cases, both humoral and cellular responses are desired. Interestingly, particulate antigen delivery systems have shown enhanced cross-MHCI presentation due to their endocytic uptake and thus may lead to both humoral and longlasting cellular responses making them ideal adjuvants [39, 40]. Interestingly, we have previously shown a strong cytotoxic T-cell and antibody response with the Ni-NPs similar to those used for comparison in the present study [25].

We are currently evaluating T-cell responses to further investigate the type of immune responses. To further improve the immune response with the Ni-NCs, we are also investigating the addition of an immunostimulant adjuvant in the Ni-NCs by either surface conjugation or incorporation in the core.

\section{Conclusions}

We have successfully formulated novel NCs with surface accessible $\mathrm{Ni}$ and investigated binding of two His-tagged proteins, GFP and Gag p41, respectively, to the surface of Ni$\mathrm{NCs}$. The Ni-NCs are non-toxic up to $24 \mathrm{~h}$ at high concentrations. Enhanced antibody responses were observed compared to $\mathrm{AH}$ at much lower doses of the antigen. Treatment with Ni-NCs resulted in a higher IgG2a/IgG1 ratio compared to AH. The Ni-NCs did not stimulate the production of systemic proinflammatory cytokines, IL-12/p40 and CCL5/ RANTES after s.c. injection. Future efforts involve conjugation of different adjuvants on the surface of the Ni-NCs in addition to the surface decoration with a protein antigen to create a system that has both immune-potentiation and efficient antigen delivery characteristics. We also plan to investigate the retention and migration of Ni-NCs from the site of administration.

\section{Acknowledgments}

The project described was supported by Award Number R01AI058842 to RJM and JW from the National Institute of Allergy and Infectious Diseases. The content is solely the responsibility of the authors and does not necessarily represent the official views of the National Institute of Allergy and Infectious Diseases or the National Institutes of Health. The authors would like to thank Dr. Robert Seder (Vaccine Research Center, NIH-NIAID) for kindly providing His-Gag p41 and Dr. Kenneth L. Rock (Department of Pathology, University of Massachusetts Medical School) for providing DC2.4 dendritic cells. The authors would also like to thank Dr. Sohrab Habibi of the Mass Spectrometry Facility, Michael Chua of the Michael Hooker Microscopy Facility, Todd Gambling of Environmental Protection Agency at the University of North Carolina at Chapel Hill for assistance with ICP-MS, confocal microscopy and TEM analyses, respectively. SW would like to acknowledge financial assistance provided by the Amgen Graduate Fellowship.

\section{List of Abbreviations}

$\begin{array}{ll}\text { AH } & \text { Aluminum hydroxide } \\ \text { His } & \text { Histidine } \\ \text { NP } & \text { Nanoparticle } \\ \text { NC } & \text { Nanocapsule }\end{array}$




\section{References}

[1]. Peek LJ, Middaugh CR, Berkland C. Nanotechnology in vaccine delivery. Adv. Drug Deliv. Rev. 2008; 60:915-928. [PubMed: 18325628]

[2]. Aline F, Brand D, Pierre J, Roingeard P, Severine M, Verrier B, Dimier-Poisson I. Dendritic cells loaded with HIV-1 p24 proteins adsorbed on surfactant-free anionic PLA nanoparticles induce enhanced cellular immune responses against HIV-1 after vaccination. Vaccine. 2009; 27:52845291. [PubMed: 19450633]

[3]. Alving CR. Liposomal vaccines: clinical status and immunological presentation for humoral and cellular immunity. Ann. N. Y. Acad. Sci. 1995; 754:143-152. [PubMed: 7625648]

[4]. Audran R, Peter K, Dannull J, Men Y, Scandella E, Groettrup M, Gander B, Corradin G. Encapsulation of peptides in biodegradable microspheres prolongs their MHC class-I presentation by dendritic cells and macrophages in vitro. Vaccine. 2003; 21:1250-1255. [PubMed: 12559806]

[5]. Ott G, Barchfeld GL, Van Nest G. Enhancement of humoral response against human influenza vaccine with the simple submicron oil/water emulsion adjuvant MF59. Vaccine. 1995; 13:15571562. [PubMed: 8578842]

[6]. Panyam J, Labhasetwar V. Biodegradable nanoparticles for drug and gene delivery to cells and tissue. Adv. Drug Deliv. Rev. 2003; 55:329-347. [PubMed: 12628320]

[7]. Klippstein R, Pozo D. Nanotechnology-based manipulation of dendritic cells for enhanced immunotherapy strategies. Nanomedicine. 2010; 6:523-529. [PubMed: 20085824]

[8]. Yan W, Jain A, O'Carra R, Woodward JG, Li W, Li G, Nath A, Mumper RJ. Lipid nanoparticles with accessible nickel as a vaccine delivery system for single and multiple his-tagged HIV antigens. HIV/AIDS Res. Palliat. Care. 2009; 1:1-11.

[9]. Duncan G, Jess TJ, Mohamed F, Price NC, Kelly SM, van der Walle CF. The influence of protein solubilisation, conformation and size on the burst release from poly(lactide-co-glycolide) microspheres. J. Control. Release. 2005; 110:34-48. [PubMed: 16225952]

[10]. Patel JD, Gandhapudi S, Jones J, O'Carra R, Woodward JG, Mumper RJ. Cationic nanoparticles for delivery of $\mathrm{CpG}$ oligodeoxynucleotide and ovalbumin: In vitro and in vivo assessment. J. Biomed. Nanotechnol. 2007; 3:97-106.

[11]. Sloat BR, Sandoval MA, Hau AM, He Y, Cui Z. Strong antibody responses induced by protein antigens conjugated onto the surface of lecithin-based nanoparticles. J. Control. Release. 2010; 141:93-100. [PubMed: 19729045]

[12]. Patel JD, O'Carra R, Jones J, Woodward JG, Mumper RJ. Preparation and characterization of nickel nanoparticles for binding to his-tag proteins and antigens. Pharm. Res. 2007; 24:343-352. [PubMed: 17180725]

[13]. Hochuli E, Dobeli H, Schacher A. New metal chelate adsorbent selective for proteins and peptides containing neighbouring histidine residues. J. Chromatogr. 1987; 411:177-184. [PubMed: 3443622]

[14]. Knecht S, Ricklin D, Eberle AN, Ernst B. Oligohis-tags: mechanisms of binding to Ni2+-NTA surfaces. J. Mol. Recognit. 2009; 22:270-279. [PubMed: 19235144]

[15]. Porath J, Carlsson J, Olsson I, Belfrage G. Metal chelate affinity chromatography, a new approach to protein fractionation. Nature. 1975; 258:598-599. [PubMed: 1678]

[16]. Lauer SA, Nolan JP. Development and characterization of Ni-NTA-bearing microspheres. Cytometry. 2002; 48:136-145. [PubMed: 12116359]

[17]. Kim JS, Valencia CA, Liu R, Lin W. Highly-efficient purification of native polyhistidine-tagged proteins by multivalent NTA-modified magnetic nanoparticles. Bioconjug. Chem. 2007; 18:333341. [PubMed: 17311440]

[18]. Platt V, Huang Z, Cao L, Tiffany M, Riviere K, Szoka FC Jr. Influence of multivalent nitrilotriacetic acid lipid-ligand affinity on the circulation half-life in mice of a liposome-attached His6-protein. Bioconjug. Chem. 2010; 21:892-902. [PubMed: 20384362]

[19]. Watson DS, Platt VM, Cao L, Venditto VJ, Szoka FC Jr. Antibody response to polyhistidinetagged peptide and protein antigens attached to liposomes via lipid-linked nitrilotriacetic acid in mice. Clin. Vaccine Immunol. 2011; 18:289-297. [PubMed: 21159923] 
[20]. Shahum E, Therien HM. Immunopotentiation of the humoral response by liposomes: encapsulation versus covalent linkage. Immunology. 1988; 65:315-317. [PubMed: 3192275]

[21]. Cooper HM, Jemmerson R, Hunt DF, Griffin PR, Yates JR 3rd, Shabanowitz J, Zhu NZ, Paterson Y. Site-directed chemical modification of horse cytochrome $\mathrm{c}$ results in changes in antigenicity due to local and long-range conformational perturbations. J. Biol. Chem. 1987; 262:1159111597. [PubMed: 2442150]

[22]. Rabinovich NR, McInnes P, Klein DL, Hall BF. Vaccine technologies: view to the future. Science. 1994; 265:1401-1404. [PubMed: 7521064]

[23]. Baylor NW, Egan W, Richman P. Aluminum salts in vaccines--US perspective. Vaccine. 2002; 20(Suppl 3):S18-23. [PubMed: 12184360]

[24]. Wang X, Uto T, Akagi T, Akashi M, Baba M. Poly(gamma-glutamic acid) nanoparticles as an efficient antigen delivery and adjuvant system: potential for an AIDS vaccine. J. Med. Virol. 2008; 80:11-19. [PubMed: 18041033]

[25]. Cui Z, Patel J, Tuzova M, Ray P, Phillips R, Woodward JG, Nath A, Mumper RJ. Strong T cell type-1 immune responses to HIV-1 Tat (1-72) protein-coated nanoparticles. Vaccine. 2004; 22:2631-2640. [PubMed: 15193389]

[26]. Dong X, Mattingly CA, Tseng M, Cho M, Adams VR, Mumper RJ. Development of new lipidbased paclitaxel nanoparticles using sequential simplex optimization. Eur. J. Pharm. Biopharm. 2009; 72:9-17. [PubMed: 19111929]

[27]. Dong X, Mattingly CA, Tseng MT, Cho MJ, Liu Y, Adams VR, Mumper RJ. Doxorubicin and paclitaxel-loaded lipid-based nanoparticles overcome multidrug resistance by inhibiting Pglycoprotein and depleting ATP. Cancer Res. 2009; 69:3918-3926. [PubMed: 19383919]

[28]. Fuchs SM, Raines RT. Arginine grafting to endow cell permeability. ACS Chem. Biol. 2007; 2:167-170. [PubMed: 17319644]

[29]. Oyewumi MO, Liu S, Moscow JA, Mumper RJ. Specific association of thiamine-coated gadolinium nanoparticles with human breast cancer cells expressing thiamine transporters. Bioconjug. Chem. 2003; 14:404-411. [PubMed: 12643751]

[30]. Maassen CB, Boersma WJ, van Holten-Neelen C, Claassen E, Laman JD. Growth phase of orally administered Lactobacillus strains differentially affects IgG1/IgG2a ratio for soluble antigens: implications for vaccine development. Vaccine. 2003; 21:2751-2757. [PubMed: 12798614]

[31]. Romagnani S. T-cell subsets (Th1 versus Th2). Ann. Allergy Asthma Immunol. 2000; 85:9-18. quiz 18, 21. [PubMed: 10923599]

[32]. Marrack P, McKee AS, Munks MW. Towards an understanding of the adjuvant action of aluminium. Nat. Rev. Immunol. 2009; 9:287-293. [PubMed: 19247370]

[33]. McKee AS, Munks MW, MacLeod MK, Fleenor CJ, Van Rooijen N, Kappler JW, Marrack P. Alum induces innate immune responses through macrophage and mast cell sensors, but these sensors are not required for alum to act as an adjuvant for specific immunity. J. Immunol. 2009; 183:4403-4414. [PubMed: 19734227]

[34]. Huang Z, Park JI, Watson DS, Hwang P, Szoka FC Jr. Facile synthesis of multivalent nitrilotriacetic acid (NTA) and NTA conjugates for analytical and drug delivery applications. Bioconjug. Chem. 2006; 17:1592-1600. [PubMed: 17105240]

[35]. Huang Z, Hwang P, Watson DS, Cao L, Szoka FC Jr. Tris-nitrilotriacetic acids of subnanomolar affinity toward hexahistidine tagged molecules. Bioconjug. Chem. 2009; 20:1667-1672. [PubMed: 19650657]

[36]. Smialowicz RJ, Rogers RR, Riddle MM, Stott GA. Immunologic effects of nickel: I. Suppression of cellular and humoral immunity. Environ. Res. 1984; 33:413-427. [PubMed: 6609070]

[37]. Buzard GS, Kasprzak KS. Possible roles of nitric oxide and redox cell signaling in metal-induced toxicity and carcinogenesis: a review. J. Environ. Pathol. Toxicol. Oncol. 2000; 19:179-199. [PubMed: 10983886]

[38]. Chikh GG, Li WM, Schutze-Redelmeier MP, Meunier JC, Bally MB. Attaching histidine-tagged peptides and proteins to lipid-based carriers through use of metal-ion-chelating lipids. Biochim. Biophys. Acta. 2002; 1567:204-212. [PubMed: 12488054]

[39]. Shen H, Ackerman AL, Cody V, Giodini A, Hinson ER, Cresswell P, Edelson RL, Saltzman WM, Hanlon DJ. Enhanced and prolonged cross-presentation following endosomal escape of 
exogenous antigens encapsulated in biodegradable nanoparticles. Immunology. 2006; 117:78-88. [PubMed: 16423043]

[40]. Chen J, Li Z, Huang H, Yang Y, Ding Q, Mai J, Guo W, Xu Y. Improved antigen crosspresentation by polyethyleneimine-based nanoparticles. Int. J. Nanomedicine. 2011; 6:77-84. [PubMed: 21289984] 


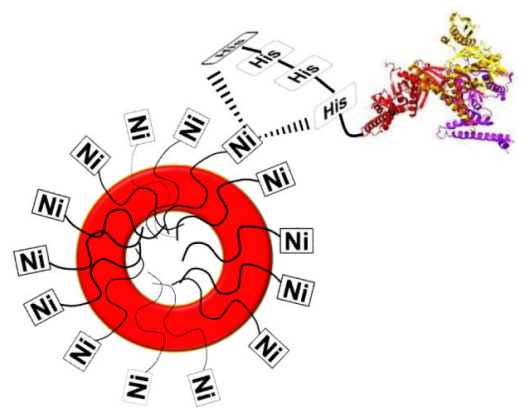

b

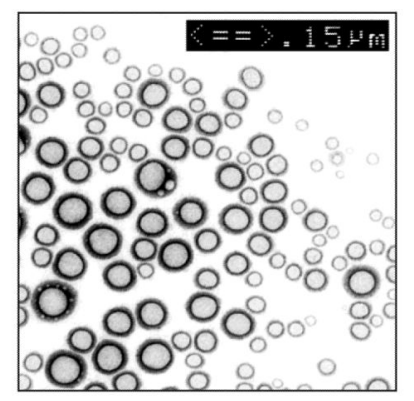

Fig. 1. Nanocapsules (NCs) with surface accessible nickel (Ni-NCs)

a) Ni-NCs having a core and shell morphology were formulated with DGS-NTA-Ni to provide surface accessible nickel for binding to His-tagged proteins. Four out of the six coordination sites of Ni in DGS-NTA-Ni are occupied while the two remaining sites are available for His-tagged ligand binding; b) TEM image of Ni-NCs. The distance between the arrowheads within the blackbox corresponds to $150 \mathrm{~nm}$. 
a

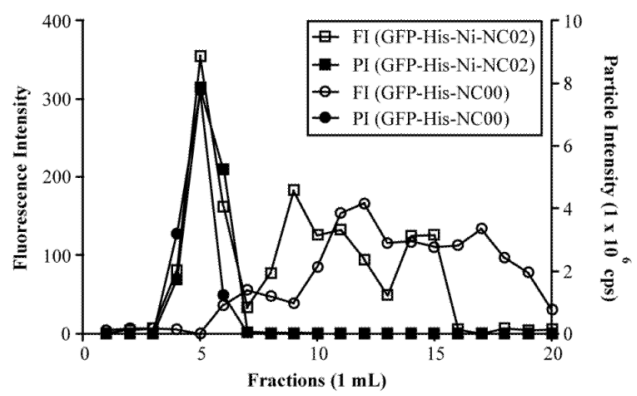

b

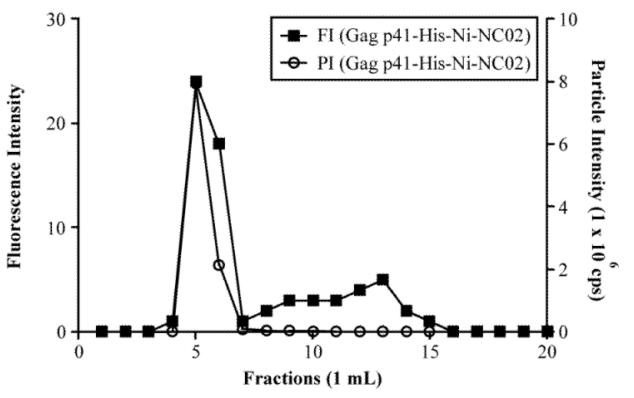

Fig. 2. Binding of His-tagged proteins to Ni-NCs

a) $\mathrm{Ni}-\mathrm{NCs}$ formulated with (formulation $\mathrm{NC02}$ ) or without (formulation $\mathrm{NCO0}$ ) surface $\mathrm{Ni}$ were compared to determine specific and non-specific binding of His-GFP as a model protein, and to determine the binding efficiency of the Ni-NCs. The Ni-NCs were separated from unbound His-GFP by elution from a Sepharose CL-4B column $(1.5 \times 15 \mathrm{~cm})$ using 10 $\mathrm{mM}$ PBS as the elution medium. The elution of Ni-NCs was monitored by measuring the particle intensity (PI) while the fluorescence intensity (FI) was measured to determine HisGFP associated with each fraction; b) Binding of fluorescein-His-Gag p41 to the surface of $\mathrm{Ni}-\mathrm{NCs}$ (formulation $\mathrm{NCO2}$ ) was determined similarly. 


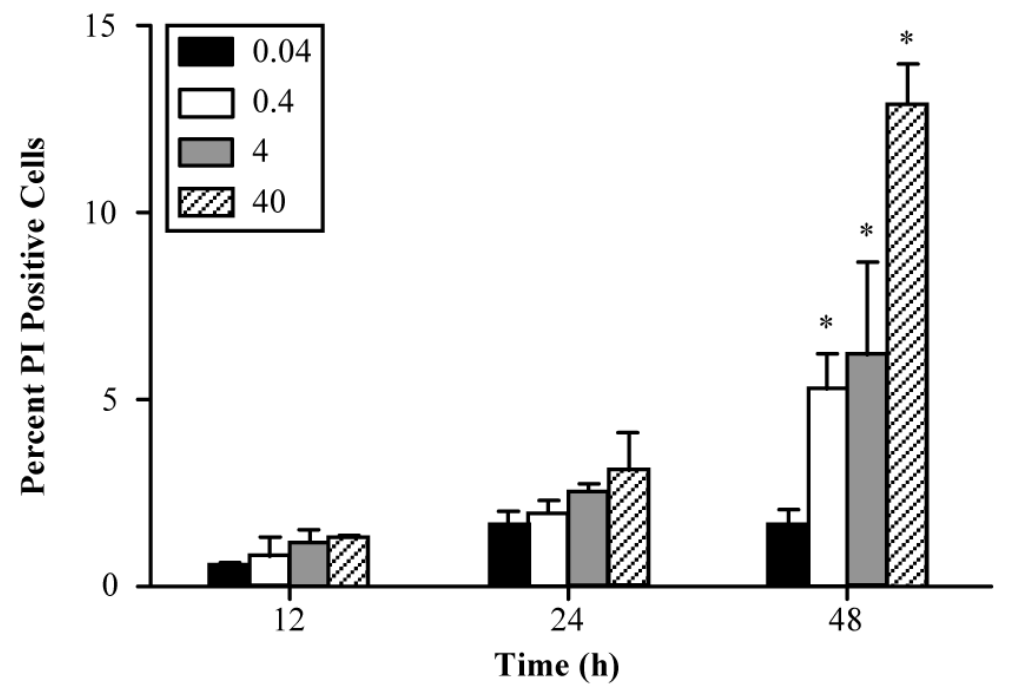

Fig. 3. Interaction of Ni-NCs with DC2.4 dendritic cells

DC2.4 dendritic cells $\left(1 \times 10^{5}\right)$ were treated with formulation NC02 $(0.04,0.4,4$ and $40 \mu \mathrm{g} /$ $\mathrm{ml}$ ) following which they were stained with propidium iodide and analyzed by flow cytometry. Data represents mean $\pm \operatorname{SEM}(\mathrm{n}=3)$. $* \mathrm{p}<0.01$. 
a

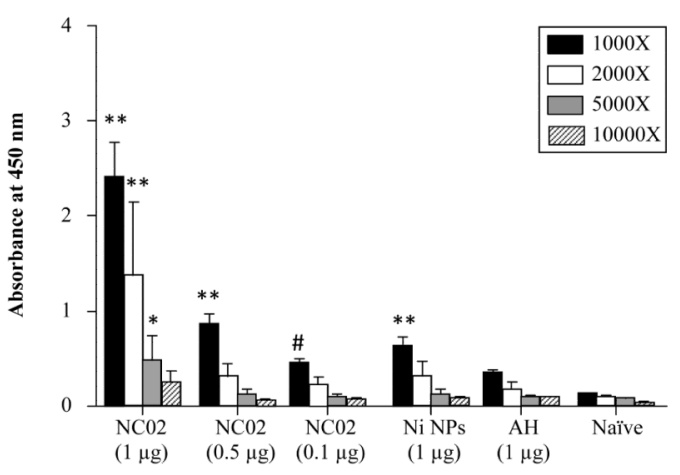

b.

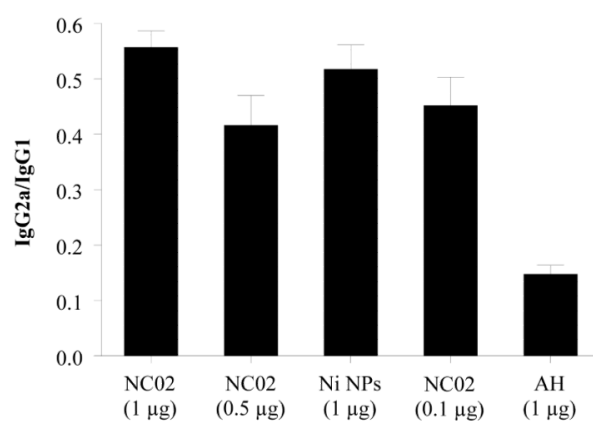

Fig. 4. Antibody responses in BALB/c mice

a). Female BALB/c mice were immunized on days 0 and 14 with $100 \mu \mathrm{L}$ of Gag p41-HisNi-NPs, Gag p41-His-Ni-NC02 or His-Gag p41 adjuvanted with Aluminum Hydroxide $(\mathrm{AH})$; b) Serum samples were also analyzed for IgG1 and IgG2a levels. His-Gag p41equivalent dose is indicated in parentheses on $\mathrm{X}$-axis. Serum was analyzed on day 28 by ELISA. Data represents mean \pm SEM $(n=6-7)$. \# $p<0.05, * p<0.01, * * p<0.001$ compared to naïve (untreated) group. 
a

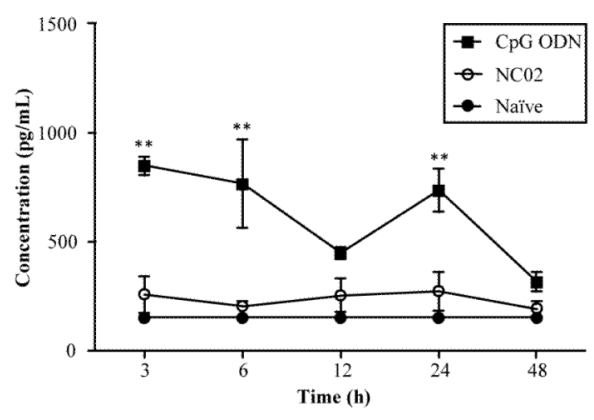

b

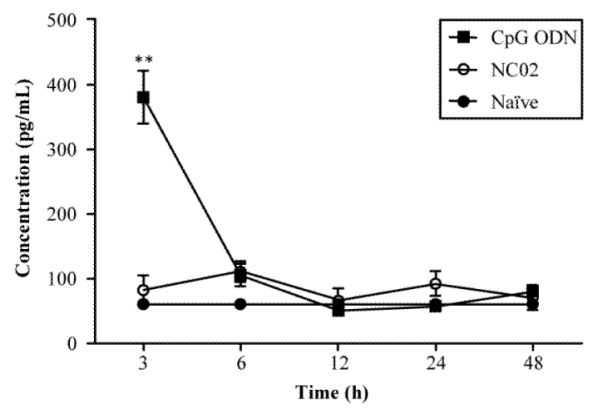

Fig. 5. Serum cytokine analysis

Serum levels of a) IL-12/p40 and b) CCL5/RANTES were measured at predetermined time points following s.c. administration of formulation NC02 $(0.48 \mathrm{mg})$ in female BALB/c mice. CpG ODN $(10 \mu \mathrm{g})$ was administered as positive control. Analysis was performed by cytometric bead array flex assay. Data represents mean $\pm \operatorname{SEM}(n=3)$, ** $\mathrm{p}<0.001$. 
Table 1

Physicochemical characterization of the representative NC formulations with or without the incorporation of surface chelated nickel.

\begin{tabular}{c|c|c|c|c}
\hline $\begin{array}{c}\text { Formulation/ } \\
\text { Parameters }\end{array}$ & NC00 & NC01 & NC02 & NC03 \\
\hline $\begin{array}{c}\text { Particle Size }(\mathbf{n m}) \\
(\text { Mean } \pm \text { SD; n=3) }\end{array}$ & $197.2 \pm 59.3$ & $199.7 \pm 67.4$ & $214.9 \pm 57.1$ & $270.2 \pm 145.2$ \\
\hline Zeta Potential $(\mathbf{m V})$ & -7.8 & -16.2 & -14.8 & -35.9 \\
\hline $\begin{array}{c}\text { Nickel Content } \\
(\text { ng/mg NPs; Mean } \pm \text { SD; n=2) }\end{array}$ & ND & $29.5 \pm 2.8$ & $145.6 \pm 19.5$ & $228.4 \pm 38.5$ \\
\hline
\end{tabular}

$\mathrm{ND}=$ Not detected 
Table 2

Estimation of accessible nickel on the surface of Ni-NCs using His-GFP as a model protein.

\begin{tabular}{c|c|c}
\hline Formulation & $\begin{array}{c}\text { Ni:GFP } \\
\text { (Molar Ratio) }\end{array}$ & $\begin{array}{c}\text { GFP Associated } \\
\text { with NCs (\%) }\end{array}$ \\
\hline NC02-01 & $1: 0.1$ & 24.87 \\
\hline NC02-02 & $1: 0.2$ & 35.68 \\
\hline NC02-03 & $1: 0.4$ & 32.28 \\
\hline NC02-04 & $1: 0.8$ & 32.70 \\
\hline NC00-01 & $0: 0.2^{*}$ & 2.34 \\
\hline
\end{tabular}

Ni-NCs were incubated with His-GFP at different molar ratios of Ni:His-GFP. The GFP-His-Ni-NCs were purified and the percentage of fluorescence associated with NCs was calculated.

Formulation NC02 was incubated with His-GFP to estimate non-specific binding in the absence of surface nickel. The amount of GFP incubated with $\mathrm{NC} 00$ was equivalent to that used in $\mathrm{NC02}$ (Ni:His-GFP of 1:0.2) trial. 


\section{Table 3}

Mouse immunization study design.

\begin{tabular}{c|c|c}
\hline Group/Dose & NCs (mg) & $\begin{array}{c}\text { His-Gag p41 } \\
\text { equivalent }(\boldsymbol{\mu g})\end{array}$ \\
\hline Naive & 0 & 0 \\
\hline Gag p41-His-Ni-NCs (NC02) & 0.48 & 1 \\
\hline Gag p41-His-Ni-NCs (NC02) & 0.24 & 0.5 \\
\hline Gag p41-His-Ni-NCs (NC02) & 0.048 & 0.1 \\
\hline AH + His-Gag p41 & 0.48 & 1 \\
\hline Gag p41-His-Ni-NPs & 0.1 & 1 \\
\hline
\end{tabular}

Female BALB/c mice were s.c. administered treatments on day 0 (prime) and 14 (boost). Sera were collected on day 28 and analyzed by ELISA. 